\title{
Seizures in Severe Malaria: Is there Direct Brain Involvement?
}

\author{
Andreas Chiabi*, ${ }^{1}$, Bolaji Obadeyi ${ }^{2}$, Seraphin Nguefack ${ }^{1}$, Joseline Zafack ${ }^{3}$, Wilfried Kouam ${ }^{3}$, \\ Pierre-Fernand Tchokoteu ${ }^{1}$, Marie-Therese Obama ${ }^{4}$, Tetanye Ekoe ${ }^{5}$ and Elie Mbonda ${ }^{1}$
}

\author{
${ }^{I}$ Yaounde Gynaeco-Obstetric and Pediatric Hospital/Faculty of Medicine and Biomedical Sciences, University of \\ Yaounde I, Cameroon \\ ${ }^{2}$ Health Logics Limited, Lagos, Nigeria \\ ${ }^{3}$ Yaounde Gynaeco-Obstetric and Pediatric Hospital, Cameroon \\ ${ }^{4}$ University Teaching Hospital/ Faculty of Medicine and Biomedical Sciences, University of Yaounde I, Cameroon \\ ${ }^{5}$ Faculty of Medicine and Biomedical Sciences, University of Yaounde I, Cameroon
}

\begin{abstract}
Seizures are a frequent manifestation of severe malaria in children especially those less than 5 years of age. Although simple febrile seizures occur in young children of the same age group, for a long time it has not been established whether seizures following severe malaria in children of this age group are a consequence of febrile illness or due to cerebral involvement.

The possibility of determining whether these seizures are simply febrile or due to cerebral involvement is important for accuracy of diagnosis and case management; it forms the basis for making prognostic assessments and predicting future neurologic outcome as part of the burden of the disease in sub-Saharan Africa. The purpose of this review is not to produce any new findings. Instead, we aim to present existing evidence for and against the possible involvement of the central nervous system in seizures accompanying cerebral malaria. This review reveals that controversy still exists and the weight of evidence seems to be on the side of a direct neurologic involvement in patients with seizures from cerebral malaria. We conclude with a call for a heightened sense of awareness and the establishment of stringent treatment regimens in patients who present with malaria complicated by seizures.
\end{abstract}

Keywords: Seizures, severe malaria, children, neurological activity, brain involvement.

\section{INTRODUCTION}

Malaria is an important cause of death and illness in children and adults in tropical countries [1]. Mortality, currently estimated at over a million per year has risen in recent years probably due to delays and inaccuracies in diagnosis as well as increasing resistance to antimalarial medicines. There were an estimated 247 million cases of malaria among 3.3 billion people at risk in 2006 , causing nearly a million deaths mostly of children below 5 years [2].

Whilst the majority of cases of malaria are mild and can be successfully treated with oral drugs, a minority may develop complicated, life threatening disease requiring parenteral treatment. Mild forms are uncomplicated and often undergo complete resolution with good prognosis. However where cases are missed, diagnosis delayed or case treatment is poor, malaria can quickly progress to life threatening forms which are known as severe malaria and are almost exclusively due to Plasmodium falciparum [3]. A paper from a hospital in Nigeria reported severe malaria accounting for a

*Address correspondence to this author at the Yaounde Gynaeco-Obstetric and Pediatric Hospital/Faculty of Medicine and Biomedical Sciences, University of Yaounde I, Cameroon, P.O. Box 4362, Yaounde, Cameroon; Tel: 002379959 8371; Fax: 002372221 24 30; E mail: andy_chiabi@yahoo.co.uk steadily increasing proportion of hospital admissions between 2000 and 2005, with nearly $13 \%$ of patients admitted having either severe malarial anaemia or cerebral malaria by the end of the study [4]. Severe falciparum malaria according to the World Health Organization (WHO) is defined as acute malaria with one or more signs of severity and/or evidence of vital organ dysfunction, while cerebral malaria is defined as severe Plasmodium falciparum malaria with cerebral manifestations, usually coma (Glasgow coma scale $<11$, Blantyre coma scale $<3$ ). Malaria with coma persisting for $>30$ minutes after a seizure is considered to be cerebral malaria [5].

Seizures are considered one of the criteria for determining the severity of malaria but it is not often known whether they occur as a result of simple febrile illness or direct involvement of the parasites in the central nervous system. The prognosis in cases of simple febrile convulsion is usually one of complete recovery while in the case of central nervous system (CNS) involvement, there are often neurological sequelae and occasionally death. The remarkable difference in the possible outcome of malaria in both instances calls for simple, case sensitive clinical criteria to enable appropriate early diagnosis and improved case treatment for reduction of morbidity and mortality from malaria. 
It is not the primary objective of this review to present any new finding. This review seeks to assess the weight of evidence for and against direct involvement of the CNS in seizures emanating from cerebral malaria and it goes on to make suggestions for expanded and improved clinical approach to case management of patients who present with seizures in cerebral malaria.

\section{SEIZURES WITHIN THE CONTEXT OF SEVERE MALARIA}

In 1990, the World Health Organization (WHO) [6] published criteria for determining the severity of malaria. These criteria were further revised in 2000 [7], 2006 [1], and recently updated in 2010 [5]. The 2010 revised criteria for severe malaria are the presence of one or more of the following: prostration, impaired consciousness, failure to feed, respiratory distress ("air hunger"), multiple seizures (more than two episodes in 24 hours), circulatory collapse, pulmonary edema (on radiological imaging), abnormal spontaneous bleeding, jaundice, hemoglobinuria, severe anemia, hypoglycemia, acidosis, renal impairment, hyperlactataemia, and hyperparasitemia.

The National Institute of Health (NIH) in 1980, defined a febrile seizure as an event in infancy or childhood, usually occurring between three months and five years of age, associated with fever but without evidence of intracranial infection or defined cause. Seizures accompanying fever in children who have suffered a previous non-febrile seizure are excluded [8]. Falciparum malaria accounted for up to $58 \%$ of illness associated with seizures in children 6 months or older in a rural Kenyan district hospital [9].

It has often been questioned and remains to be determined whether seizures in malaria are the direct consequence of CNS involvement or are simply febrile seizures. While simple febrile seizures have been documented to undergo complete resolution, multiple seizures have been shown to be a risk factor for persisting neurological and cognitive impairments following cerebral malaria [10].

A genetic component is incriminated in the etiology of seizures occurring in $2-9 \%$ of patients with viral infections (adeno viruses, respiratory syncytial viruses, enterovirus, influenzae and parainfluenzae viruses [11]. In developing countries there are few studies on the pathogenesis of seizures in malaria. However in a recent study of 325 children prospectively evaluated in Cameroon, malaria was the leading cause of febrile seizures $(67.7 \%)$, followed by ear-nosethroat and respiratory tract infections responsible for febrile seizures in $14.1 \%$ and $9.8 \%$ respectively of the children [12].

A subject of equal controversy is the pathophysiologic mechanism of the involvement of the CNS in cerebral malaria. Various mechanisms have been elucidated to explain cerebral involvement in malaria [13-18], with some studies demonstrating evidence of cerebral involvement in the pathogenesis of seizures and a few others presenting arguments against. The hallmark of cerebral involvement in malaria is engorgement of cerebral capillaries and venules [13]. In most fatal cases, the post mortem brain is oedematous, with petechial hemorrhages and several larger brain hemorrhages [19]. The following pathophysiologic mechanisms have been hypothesized to explain cerebral involvement in patients with cerebral malaria:

- Sequestration: trapping of erythrocytes containing mature forms of the parasite in the vasculature of vital organs.

- Cytoadherence: specific interaction between PRBCs and the vascular endothelium.

Rosetting and agglutination: the adherence of non parasitized RBCs (NPRBCs) to parasitized RBCs (PRBCs) (rosetting) and PRBCs to PRBCs (agglutination).

- $\quad$ Red cell deformability: as the parasite grows within the RBC, the erythrocyte becomes deformable and this may contribute to red cell destruction and impairment of microcirculatory flow.

- Cytokines: blood concentrations of pro inflammatory cytokines are raised in cerebral malaria. Tumor necrosis factor-alpha (TNF- $\alpha$ ) upregulates endothelial cytoadherence receptors and causes hypoglycaemia and dyserythropoiesis which are features of severe disease. It has also been suggested that TNF- $\alpha$ induces the release of nitric oxide (NO) which interferes with synaptic transmission, causing coma [14, $15,20,21]$.

After a thorough search and review of medical literature no studies were found that demonstrate cerebral changes following febrile seizures. Determining whether seizures in severe malaria are simply febrile or due to cerebral involvement would be an important development for improving accuracy of diagnosis and case management. It forms the basis for making prognostic assessments and predicting future neurologic outcome as part of the burden of the disease in sub-Saharan Africa. In order to establish the extent to which one or more of these pathologic changes and pathways account for seizures in children with cerebral malaria, we conducted a review of relevant papers available on this topic from PubMed and Medline.

\section{CURRENT EVIDENCE FOR OR AGAINST DIRECT INVOLVEMENT OF THE PARASITES ON THE CNS}

The malaria parasite has been implicated in pathological changes observed in the CNS of children with cerebral malaria. In some cases, a strong causal relationship has been established between the neuropathologic changes induced by the parasite in the brain and the occurrence of seizures in patients with cerebral malaria (CM). Such studies include, those associating fever and / or levels of parasitaemia in the brain to the occurrence of seizures. Others demonstrate neuropathologic observations which range from parasite induced apoptosis, the finding of angiogenic factors, reduced levels of neuroprotective factors to changes in cerebrospinal fluid markers as evidence of cellular changes and cellular malfunction. Additional changes observed at post-mortem relate to platelet densities, platelet aggregation within microvasculature and aberrant platelet function. The use of imaging techniques also lends support for cerebral involvement in malaria with seizures.

Armah et al. [22] did a post mortem study of cerebrospinal fluid (CSF) and serum biomarkers of apoptosis and angi- 
ogenesis, inflammatory indicators including cytokines and chemokines in 19 cadavers of Ghanaian children. Their observations support the implication of parasite-induced apoptosis and angiogenic factors in CM neuropathology. Investigating 9 subjects categorized as having died of cerebral malaria $(\mathrm{CM}), 5$ subjects from malaria complicated by severe anemia/ severe malarial anemia (SMA), and another 5 subjects as non-malaria (NM) deaths; the study demonstrated an association between cerebral malaria and convulsions due to direct involvement of the parasite in the CNS. The exact mechanism of this involvement is still obscure. It was also able to link mortality with elevated serum and CSF levels of apoptotic factors (IP-10, IL-1ra, sTNFR2, sFas) with reduced serum and CSF levels of neuroprotective angiogenic growth factors (PDGFbb). All $9 \mathrm{CM}$ cases investigated had either seizures, hypertonicity or posturing of limbs. At autopsy, the $\mathrm{CM}$ cases had a slate gray discoloration of the brain, white matter petechial hemorrhages in the brain, and/or erythrocytes, and/or malaria pigment in the cerebral microvasculature, and none of these in the SMA and NM groups. As would be expected, the parasite density was significantly higher in the SMA group than in the CM and NM groups. Platelet levels were statistically significantly lower in the CM group, than in the SMA and NM groups at 170800,284 000 and $330700 / \mu \mathrm{L}$ respectively. Platelet derived growth factor is a key factor that mediates vascular smooth muscle cell proliferation and serves a neuroprotective role by inducing regeneration of damaged axons and neuronal growth after ischemia.

Akpede et al. [23] in a study of 446 Nigerian children 6 months to 5 years old with severe malaria, showed that seizures in malaria are more often a manifestation of cerebral dysfunction rather than just being simply febrile in etiology. This study demonstrated a statistically significant relationship $(\mathrm{P}<0.001)$ between parasitaemia, coma, age, pattern of convulsions, hematocrit and blood sugar; and between the severity of parasitemia and risk of convulsions, prevalence of hepatosplenomegaly and severe malaria. It then concluded that all forms of cerebral dysfunction in malaria including repeated convulsions should be managed as cerebral malaria.

Medana et al. [24] in a retrospective study of CSF markers (on 143 CSFs) of brain parenchyma damage in Kenyan children with severe falciparum malaria demonstrated a correlation between high $\mathrm{S}-100 \mathrm{~B}$ levels and recurrence of seizures during the course of admission thus lending strength to the conclusion that seizures may indeed be caused by cerebral dysfunction. A weaker correlation was found between high tau levels and seizure recurrence. Tau is a phosphorylated microtubule associated protein, considered to be important for maintaining the stability of axonal microtubules involved in the mediation of fast axonal transport of synaptic constituents. Tau levels were found to be associated with duration of coma and S-100B was associated with seizures. $\mathrm{S}-100 \mathrm{~B}$ is an acidic calcium-binding protein synthesized in astrocytes in all parts of the central nervous system.

Idro et al. [25], in a similar retrospective study of medical records of 19560 children less than 14 years $(33.6 \%$ of admissions) admitted for malaria in the Kilifi District hospital from 1992 to 2004, observed that seizures were recorded in $37.5 \%$ of those with severe malaria. Seizures in this study, were associated with parasitemia but not fever as further support that cerebral involvement was likely etiology of seizures. Although neurologic involvement was observed at low levels of parasitemia, the proportion with neurological involvement increased with rising parasitemia. Consequently, neurologic involvement was present in $40 \%$ of patients with parasite densities lower than 100,000 per $\mu \mathrm{L}$, in $50 \%$ of patients with densities between 100,000 and 500,000 per $\mu \mathrm{L}$, and in more than $60 \%$ of those with densities higher than $1,000,000$ per $\mu \mathrm{L}\left(\mathrm{x}^{2}\right.$ for trend $\left.=120 ; \mathrm{P}<0.001\right)$. These findings also indicate involvement of Plasmodium falciparum on the CNS lending credence to the fact that falciparum is often the specie responsible for cerebral malaria and its sequelae.

Apart from being the commonest illness associated with seizures, studies show that malaria has a statistically significant association with the highest seizure frequency and the occurrence of status epilepticus in children 6 months or older [9]. In a prospective study, 900 out of 4,921 children 0-13 years admitted in the Kilifi hospital in Kenya over 2 years experienced acute seizures [9], giving an incidence of acute seizures of 425 per 100,000 admissions/year and 879 per $100,000 /$ year in children less than 5 years. Plasmodium falciparum malaria was the commonest cause of seizures in $58 \%$ of the children. The proportion of children with seizures and a positive malaria slide $(55.3 \%)$ was higher and statistically significant at $\mathrm{P}<0.001$, when compared to those without seizures $(16.5 \%)$. The risk of seizures was thought to rise with increasing parasite density. Children who experienced two or more seizures had a higher geometric mean parasite density $(34,167$ [ 95\% [CI $25,910-45,054] / \mu \mathrm{L}) \mathrm{com}-$ pared to those with single or no seizures $(23,962$ [95\% CI 20,135-28,517]/ $\mu \mathrm{L}, \mathrm{p}<0.001)$.

A retrospective study by Medana et al. [26] confirmed that brain parenchymal damage is present at the time of admission in patients with severe malaria and axons are the cellular component most severely affected. This study analysed by immunoassay, three cerebrospinal fluid markers of brain parenchymal damage in 20 Vietnamese adults. For degenerated axons the study evaluated microtubuleassociated protein tau; for neurons it analysed neuron specific enolase (NSE) and S100B for astrocytes. The mean concentration of tau proteins in the CSF was significantly raised in patients with severe malaria compared with controls $(\mathrm{P}=0.0003)$. By contrast, the mean concentration of NSE and S100B remained within the normal range. Tau levels were linearly associated with duration of coma $(\mathrm{P}=0.004)$ and $\mathrm{S} 100 \mathrm{~B}$ was associated with convulsion $(\mathrm{P}=0.006)$.

This axon vulnerability in severe malaria was also demonstrated by Medana et al. in 2002 [27] by immunochemistry of brain sections in Vietnamese adult patients who died of falciparum cerebral malaria.

The vulnerability of the neural axon in malarial patients was further demonstrated by quantitative $\beta$ amyloid precursor protein ( $\beta$-APP) immunochemistry in brain sections. $\beta$ APP is a protein that is normally transported along the axon, and accumulates at sites of axonal injury. The frequency and extent of $\beta$-APP staining were more sev in cerebral malarial patients than in those without cerebral involvement. $\beta$-APP was often associated with hemorrhages and areas of demyelination suggesting that multiple processes may be involved in neuronal injury. Disruption in axonal transport in these patients may represent a final common pathway leading to 
neurological dysfunction with seizures. Axonal injury disrupts neuronal integrity, the distribution of neurosecretory granules, and the transport of enzymes and chemicals involved in the formation of neurotransmitters and substances associated with trophic activity.

Using radiologic or other imaging techniques, there are case reports of cerebral lesions in adult patients with cerebral malaria associated with seizures. Central venous thrombosis has been noted in patients using magnetic resonance imaging (MRI) and magnetic resonance venography (MVR) [28], and on autopsy and angiography in some cases [29]. Lesions of the splenium of the corpus callosum and cortical infarcts have also been noted in a patient with cerebral malaria with coma and seizures [30]. All these demonstrate cerebral involvement, in adult patients with malaria associated with seizures. Whether the same changes occur in children with cerebral malaria is not known.

Below are hypothetical mechanisms involved in the genesis of seizures by, direct action of the parasite in the CNS (Fig. 1) and by indirect action through hypoxia gener- ated by the parasite in the blood (Fig. 2); and morphological and biochemical consequences from the action of the parasite in the CNS (Fig. 3).

Relevant literature against direct involvement of the parasite on the CNS in the genesis of seizures in patients with cerebral malaria is relatively rare, and unfortunately we may not be able to give a balanced discourse or equity in this controversy.

However, Waruiru et al. [31] reviewed the files of 1324 children admitted in the pediatric ward of the Kilifi District hospital in Kenya, over a one year period and found seizures in $15.8 \%$ of children as part of their illness, with malaria accounting for $69 \%$ of the causes of seizures. Most seizures occurred between the ages of 6 months and 5 years and $64.3 \%$ of malaria-associated seizures occurred greater than two years old. There was no relationship between temperature on admission and occurrence of seizures in children with either malaria or other diagnoses. There was no relationship between parasite densities and presence of seizures in children with a primary diagnosis of malaria. In those with

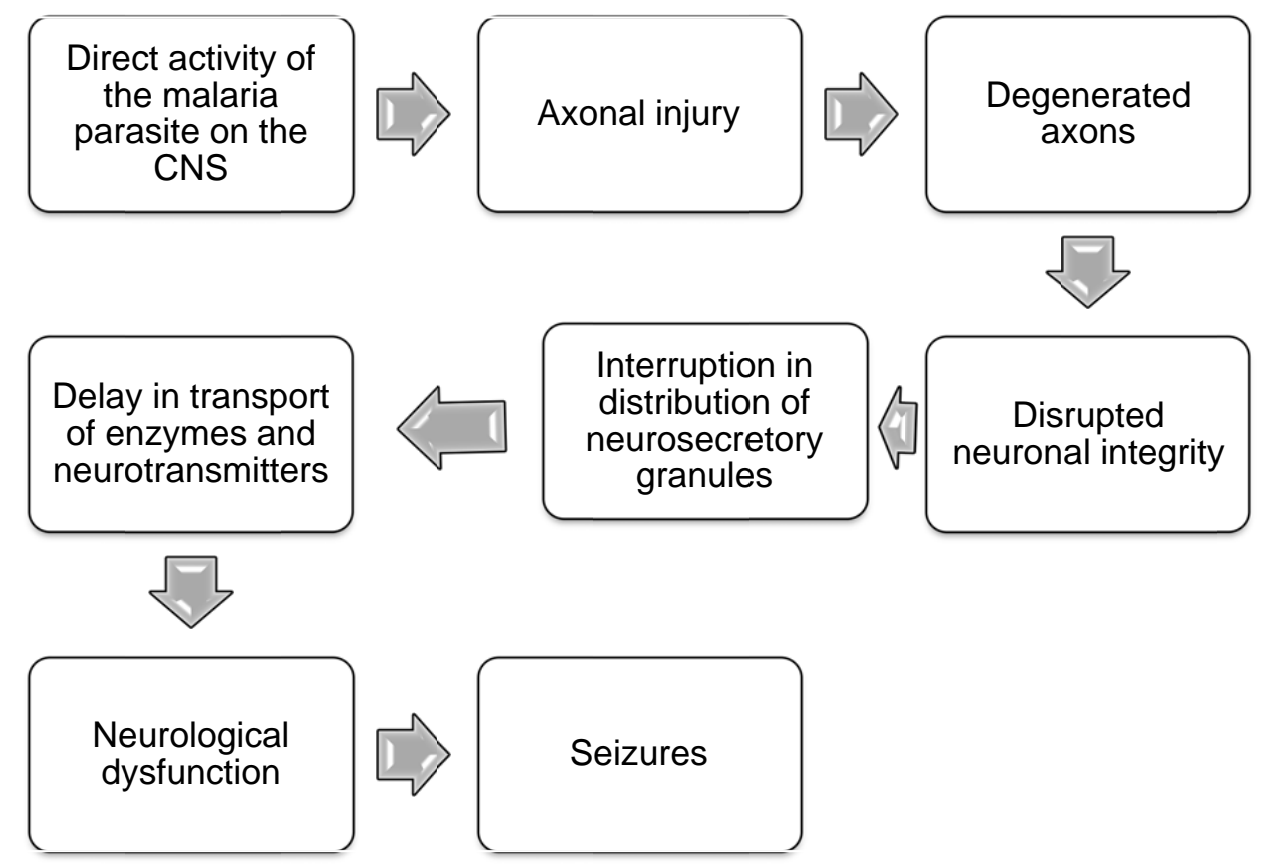

Fig. (1). Hypothesized axon vulnerability in severe malaria.

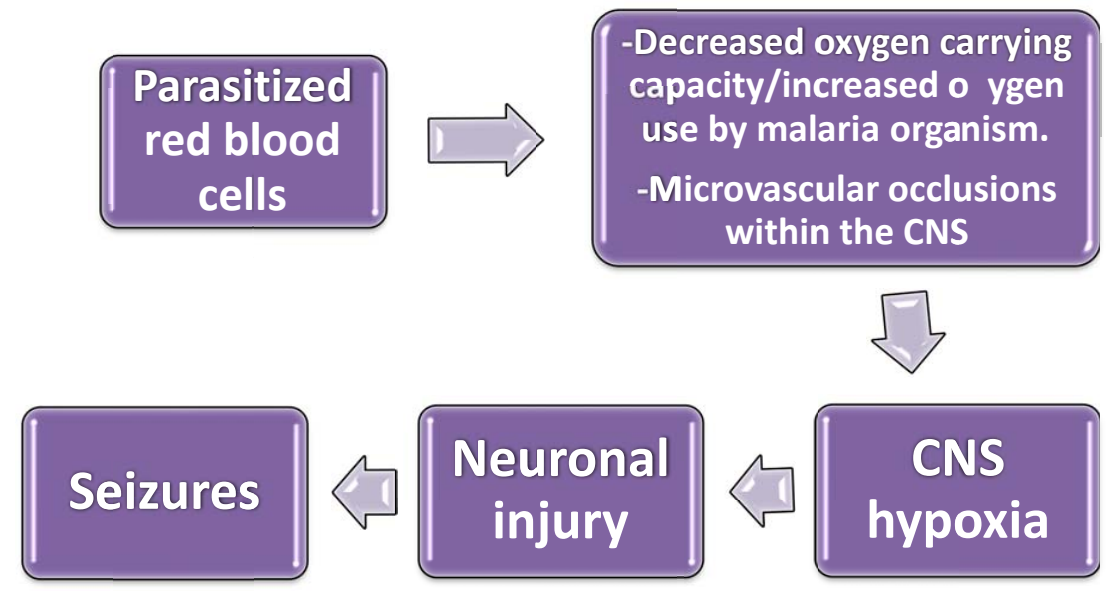

Fig. (2). Hypothesized genesis of seizures from hypoxia caused by the malaria parasite. 


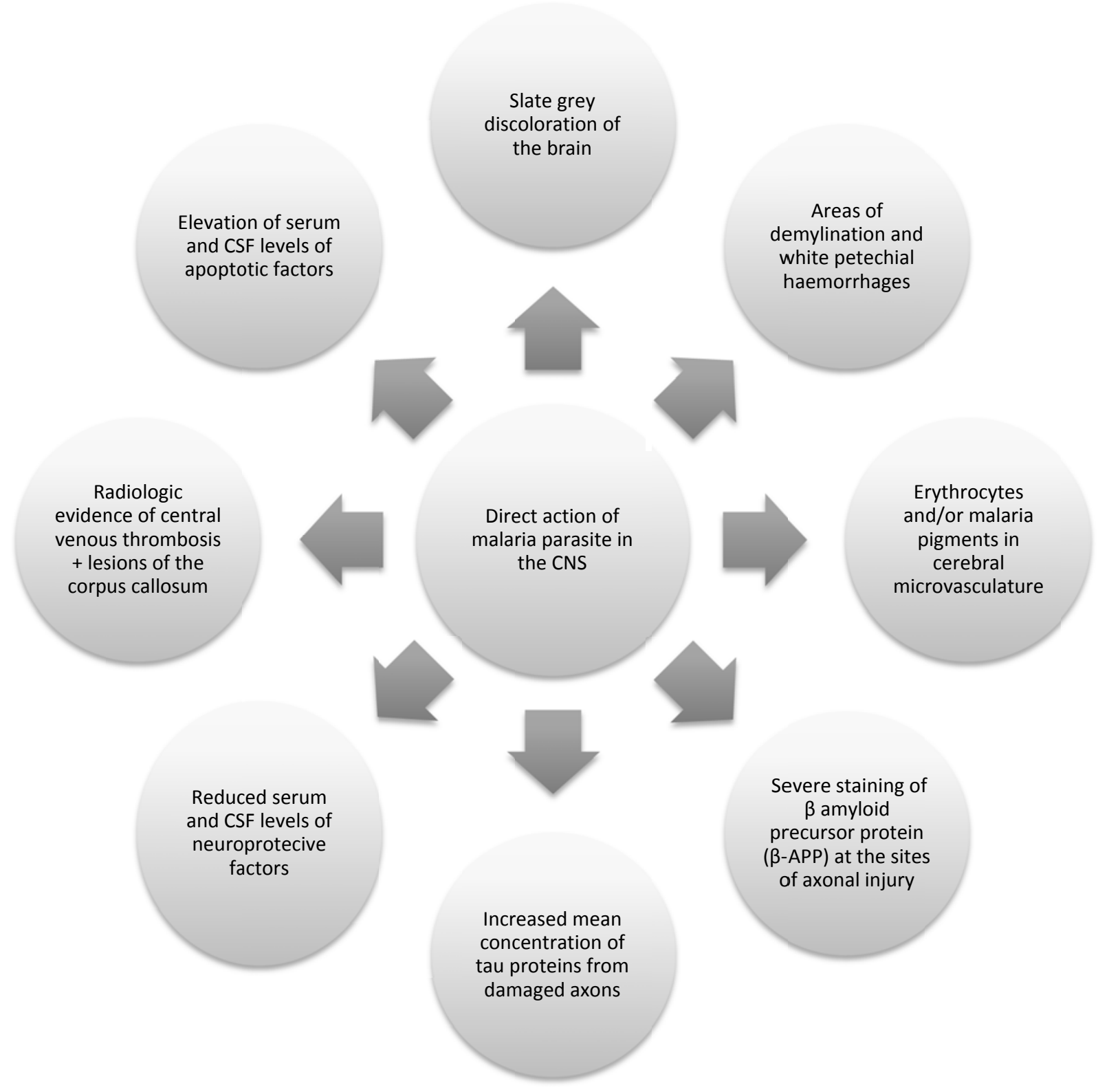

Fig. (3). Hypothesized morphologic and biochemical consequences from the direct action of the malaria parasite on the CNS.

non malaria diagnoses, there was a higher proportion with a past history of seizures than those with parasitemia.

Nguefack et al. [12] in a prospective study of 325 children admitted in a referral hospital in Yaounde, Cameroon with febrile convulsions, found no significant relationship between parasite density and the risk of febrile seizures.

\section{CONCLUSION}

The burden of malaria is finally receiving the attention it deserves with the scaling up of preventive interventions such as insecticide treated nets (ITNs), increased accuracy of diagnosis and improvement in case management with the adoption of treatment protocols globally. Unfortunately, cerebral malaria continues to be the most severe and deadly form of the disease. This review posed the question - do seizures in children with cerebral malaria carry the favorable prognosis of simple febrile seizures or are they a sign of di- rect neurologic involvement with poorer prognosis? Although simple febrile seizures have been known to occur in patients with malaria, the weight of evidence seems to support a direct neurologic involvement in patients with seizures from cerebral malaria. While febrile seizures often resolve uneventfully, seizures from cerebral malaria involving the scope of neuropathologic changes discussed in this review represent a poor prognostic sign and are often followed by neurologic sequelae.

From a preventive perspective, current public health efforts at prevention through the use of ITNs and indoor spraying techniques need to be sustained and further intensified. Urgently required is increased availability of accurate malaria diagnostic tools and the Artemisinin Combined Treatment (ACT) particularly in the remote areas of Africa where most malaria deaths occur. 
As CM is most often an escalation of simple malaria into a life threatening form, health workers need to adopt a proactive approach to arrest this progression especially in the susceptible population of children under 5 years of age who present with simple uncomplicated malaria. In essence, there is a need to adopt a heightened sense of awareness and establish an aggressive treatment regimen in managing patients who present with malaria especially when complicated with seizures. This, we believe, will go a long way in further reducing morbidity and mortality from malaria infection in general and cerebral malaria in particular.

From this review, there appears to be no unanimity as to direct brain involvement in the genesis of seizures in cerebral malaria. Our conclusion is in line with the WHO recommendation that a patient with any degree of impaired consciousness or other signs of cerebral dysfunction be treated with the utmost urgency, and we believe this attitude should be adopted especially in malaria endemic areas.

We hope that this initial attempt to review the prognostic and pathophysiologic implications of seizures in cerebral malaria will encourage more detailed work or systematic reviews in this important field of study. Such basic, applied or operational research may identify the exact mechanism of effect of the malaria parasite on the CNS, and therefore target treatment to specific aspects of the pathology within the brain.

\section{REFERENCES}

[1] World Health Organization. Guidelines for the treatment of malaria. WHO/HTM/MAL/2006.1108

[2] World Health Organization. World Malaria Report. WHO/HTM/ GMP/2008.1

[3] Pasvol G. The treatment of complicated and severe malaria. $\mathrm{Br}$ Med Bull 2006; 75: 29-47.

[4] Orimadegun AE, Fawole O, Okereke JO, Akinbami FO, Sodeinde O. Increasing burden of childhood severe malaria in a Nigerian tertiary hospital: implication for control. J Trop Pediatr 2007; 53: 18589.

[5] World Health Organization. Guidelines for the treatment of malaria. $2^{\text {nd }}$ ed 2010.

[6] World Health Organization. Severe and complicated malaria. Trans R Soc Trop Med Hyg 1990; 84(2): 1-65.

[7] World Health Organization. Severe falciparum malaria. Trans R Soc Trop Med Hyg 2000; 94(Suppl 1): 51-90.

[8] National Institutes of Health. Consensus Development Conference Statement. May 19-21, 1980. Febrile seizures. http://consensus.nih. gov/1980/1980FebrileSeizures023html.htm. [Accessed 6 April 2010].

[9] Idro R, Gwer S, Kahindi M, et al. The incidence, aetiology and outcome of acute seizures in children admitted to a rural Kenyan district hospital. BMC Pediatr 2008; 8: 5 .

[10] Idro R, Carter JA, Fegan G, Neville BGR, Newton CRJC. Risk factors for persisting neurological and cognitive impairments following cerebral malaria. Arch Dis Child 2006; 91: 142-8.
[11] Auvin S, Vallée L. Connaissances actuelles sur les mécanismes physiopathologiques des convulsions fébriles. Arch Pediatr 2009; 16: 450-6.

[12] Nguefack S, Ngo Kana CA, Mah E, et al. Aspects cliniques, étiologiques et thérapeutiques des convulsions fébriles. A propos de 325 cas à Yaounde. Arch Pediatr 2010; 17: 480-5.

[13] Newton RJC, Hien TT, White N. Cerebral malaria. J Neurol Neurosurg Psychiatry 2000; 69: 433-41.

[14] Dondorp AM. Pathophysiology, clinical presentation and treatment of cerebral malaria. Neurol Asia 2005; 10: 67-77.

[15] Newton RJC, Taylor TE, Whitten RO. Pathophysiology of fatal falciparum malaria in African children. Am J Trop Med Hyg 1998; 58: 673-83.

[16] Miller LH, Baruch DI, Doumbo OK. The pathogenic basis of malaria. Nature 2002; 415: 673-9.

[17] Mackintosh CL, Beeson JG, Marsh K. Clinical features and pathogenesis of severe malaria. Trends Parasitol 2004; 20: 597-603.

[18] Dondorp AM, Pongponratn E, White NJ. Reduced microcirculatory flow in severe falciparum malaria: pathophysiology and electronmicroscopic pathology. Acta Tropica 2004; 89: 309-17.

[19] Department of Tropical Pathology, Faculty of Tropical Medicine, Mahidol University. Pathology and electron microscopy in severe malaria. www.tm.mahidol.ac.th/eng/tmpt/tmpt_malaria_ppt.pdf [Accessed 5 April 2010].

[20] Medana IM, Turner GDH. Plasmodium falciparum and the blood brain barrier-contact and consequences. J Infect Dis 2007; 195: 921-3.

[21] Medana IM, Turner GDH. Human cerebral and the blood-brain barrier. Int J Parasitol 2006; 36: 555-68.

[22] Armah HB, Wilson NO, Sarfo BY, et al. Cerebrospinal fluid and serum biomarkers of cerebral malaria mortality in Ghanian children. Malar J 2007; 6: 147.

[23] Akpede GO, Sykes RM, Abiodun PO. Convulsions with malaria: Febrile or indicative of cerebral involvement? J Trop Pediatr 1993; 39: 350-5.

[24] Medana IM, Idro R, Newton CR. Axonal and astrocyte injury markers in the cerebrospinal fluid of Kenyan children with severe malaria. J Neurol Sci 2007; 258: 93-8.

[25] Idro R, Ndiritu M, Ogutu B, et al. Burden, features, and outcome of neurological involvement in acute falciparum malaria in Kenyan children. JAMA 2007; 297: 2232-40.

[26] Medana IM, Lindert RB, Wurster U, et al. Cerebrospinal fluid levels of markers of brain parenchymal damage in Vietnamese adults with severe malaria. Trans R Soc Trop Med Hyg 2005; 99: 610-7.

[27] Medana IM, Day NP, Hien TT, et al. Axonal injury in cerebral malaria. Am J Pathol 2002; 160: 655-66.

[28] Luvira V, Chamnanchanunt S, Thanachartwet V, Phumratanaprapin W, Viriyavejakul A. Cerebral venous sinus thrombosis in severe malaria. Southeast Asian J Trop Med Public Health 2009; 40: 893-7.

[29] Krishnan A, Karnad DR, Limaye U, Siddharth W. Cerebral venous and dural sinus thrombosis in severe falciparum malaria. J Infect 2004; 48: 86-90.

[30] Hantson P, Hernalsteen D, Cosnard G. Reversible splenial lesion syndrome in cerebral malaria. J Neuroradiol 2010; 37(4): 243-6.

[31] Waruiru CM, Newton CR, Forster D, et al. Epileptic seizures and malaria in Kenyan children. Trans R Soc Trop Med Hyg 1996; 90: $152-5$. 